\title{
Seeking the heart, brain and nerve in Oz: moving Internationalism beyond a cloak for commercial dominance or intellectual snobbery
}

\author{
Brian Shackel, \\ Founder Director, HUSAT Research Institute, \\ Loughborough University, U.K.
}

In a more famous journey to $\mathrm{Oz}$, the travellers' quest was for a heart, a brain and 'the nerve'. This panel explores a parallel contemporary quest in the field of $\mathrm{HCI}$ from an international perspective.

In the quest for market dominance, there is a risk that computer companies will only do enough to internationalise software to be able to say that "we have an international product so you should buy it". In comparison with what COULD be done, current efforts to internationalise seem tokenistic at best and patronising at worst---i.e. $\mathrm{HCI}$ needs to move in a heartfelt way beyond effort which could be characterised as "we'll let you understand the language (else you won't buy the product), but we're not too interested in your customs and culture".

\begin{abstract}
Such a development needs to re-examine the way that western society thinking (brain) tends to equate 'more' with 'better', proving slow to learn about the importance of intermediate and ultrasimple technology. Other parts of the world hold the concept of 'appropriate technology' where technology does not become an end in itself, and where more 'bells and whistles' are not good but bad technology. Rather, there is an argument that it is time for the western computing industry to realise that people do not NEED heaps of functionality as they only ever use a small proportion of it.

To adopt such a quest requires nerve---and demonstrable benefits. This panel will discuss some of the benefits to be achieved through internationalism, and will include an Australian business perspective.
\end{abstract}

